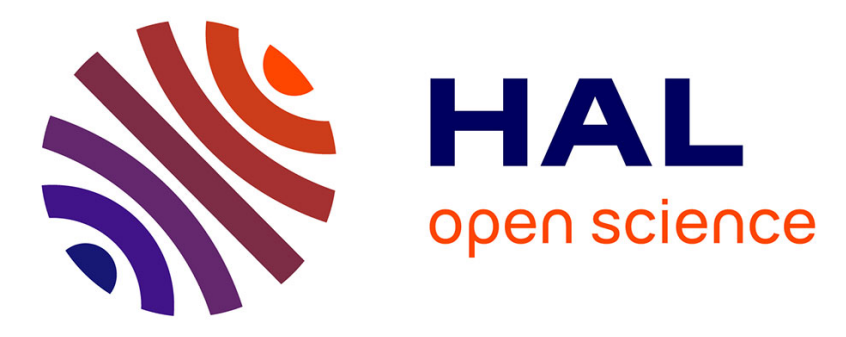

\title{
Parametric generation of twin photons in vertical triple microcavities
}

Carole Diederichs, Taj David, Thimotée Lecomte, Cristiano Ciuti, Philippe

Roussignol, Claude Delalande, Jérôme Tignon, Aristide Lemaitre, Ludovic

Largeau, Olivia Mauguin, et al.

\section{To cite this version:}

Carole Diederichs, Taj David, Thimotée Lecomte, Cristiano Ciuti, Philippe Roussignol, et al.. Parametric generation of twin photons in vertical triple microcavities. Comptes Rendus. Physique, 2007, 8 (10), pp.1198. hal-00262215

\section{HAL Id: hal-00262215 \\ https://hal.science/hal-00262215}

Submitted on 21 Mar 2008

HAL is a multi-disciplinary open access archive for the deposit and dissemination of scientific research documents, whether they are published or not. The documents may come from teaching and research institutions in France or abroad, or from public or private research centers.
L'archive ouverte pluridisciplinaire HAL, est destinée au dépôt et à la diffusion de documents scientifiques de niveau recherche, publiés ou non, émanant des établissements d'enseignement et de recherche français ou étrangers, des laboratoires publics ou privés. 


\title{
Parametric Generation of Twin Photons in Vertical Triple Microcavities
}

\author{
Carole Diederichs, David Taj, Thimothée Lecomte, Crisitiano Ciuti, \\ Philippe Roussignol, Claude Delalande, Jérôme Tignon \\ Laboratoire Pierre Aigrain, Ecole Normale Supérieure, 24 rue Lhomond, F-75005 Paris, \\ France
}

Aristide Lemaître, Ludovic Largeau, Olivia Mauguin, Jacqueline Bloch

LPN-CNRS, Route de Nozay, F-91460 Marcoussis, France

Charles Leyder, Alberto Bramati, Elisabeth Giacobino

Laboratoire Kastler Brossel, Université Paris 6, Ecole Normale Supérieure, F-75005, France

\begin{abstract}
We report the realization of a monolithic vertical-cavity, surface emitting micro-optical parametric conversion nanostructure, triply resonant with the parametric frequencies, allowing parametric oscillation with ultra-low pump power threshold. The photonic phasespace naturally provides triple resonance for the parametric frequencies, together with builtin cavity phase-matching for the pump wave at normal incidence. Parametric oscillation is observed in both the strong and weak exciton-photon coupling regime, allowing high operating temperature. Signal and idler beams can be collected at $0^{\circ}$ or at finite angles. The OPO threshold is low enough to envisage the realization of an all-semiconductor electrically-pumped micro-parametric oscillator.
\end{abstract}

Key words: optical parametric oscillation, semiconductors, microcavity, twin photons Corresponding author: J. Tignon

Email: jerome.tignon@lpa.ens.fr; Phone: + (33) 144323354 ; Fax: + (33) 144323840

\section{Introduction}

Optical parametric oscillation (OPO) is a nonlinear process that enables coherent generation of signal and idler waves, shifted in frequency from the pump wave. Efficient parametric conversion is the paradigm for the generation of twin or entangled photons for quantum optics applications such as quantum cryptography [1], or for the generation of new frequencies in spectral domains not reachable by existing devices. Rapid development in 
the field of quantum information requires monolithic, alignment free sources allowing for efficient coupling into optical fibers and possibly electrical injection. During the last decade, many efforts have been devoted to the development of integrated devices for quantum information and for realizing all-semiconductor parametric oscillators. Nevertheless, to date OPOs typically rely on nonlinear crystals placed into complex external cavities, and pumped by powerful external lasers. Long interaction lengths are typically required and the phase mismatch between the parametric waves propagating at different velocities results in poor parametric conversion efficiencies. Recently $[2,3,4]$, it has been demonstrated that very large $\chi^{(3)}$ polaritonic nonlinearities in semiconductor microcavities can be used to achieve low-threshold optical parametric oscillation. In conventional planar microcavities, the phase-matching is obtained thanks to the strong coupling of excitons and photons that leads to an S-shaped low-energy polariton dispersion. After excitation at a well-defined angle (sometimes referred to as the magic-angle), two pump-polaritons can be efficiently scattered, one to low energy at $0^{\circ}$ (signal), and one to high energy and larger angle (idler). This has several important drawbacks: working temperatures are very limited (the strong coupling is mandatory); the system must be optically pumped at a large angle; last, the idler is emitted at an even larger angle and only weakly coupled to light, precluding efficient collection for quantum optics applications.

Here, we report the realization of a monolithic vertical-cavity, surface emitting micro-optical parametric conversion nanostructure, triply resonant with the parametric frequencies, allowing parametric oscillation with ultra-low pump power threshold $[5,6,7]$. The photonic phase-space naturally provides triple resonance for the parametric frequencies, together with built-in cavity phase-matching for all waves at normal incidence. This allows easy pumping and collection of the signal and idler at $0^{\circ}$. Alternatively, we show that the signal and idler beams can be collected efficiently at finite angles. The OPO geometry and low threshold allow to envisage the realization of an all-semiconductor electrically-pumped micro-parametric oscillator.

\section{Sample description}

The scheme of the MBE grown triply resonant microcavity nanostructure is display in Figure 1. It consists of three $\lambda$-GaAs microcavities $(840 \mathrm{~nm})$, coupled via two intermediate Bragg mirrors (DBR1 and DBR2). The ensemble is enclosed between a bottom and a top DBR. The active medium consists of a set of quantum wells (QWs) at the anti-nodes of the electromagnetic field. In the following, we refer to two samples showing distinct thresholds. Sample A (resp. sample B) consists of 13 pairs of AlAs/GaAs in each Bragg mirror (resp. 15, 11, 11 and 18 pairs) and one $\mathrm{In}_{0.05} \mathrm{Ga}_{0.95} \mathrm{As} \mathrm{QW}$ in each cavity (resp. 9 QWs). The cavity finesse is 740 for sample A and 1350 for sample B. A slight wedged has been voluntarily introduced in all three resonators so that the cavity-mode energies can be independently adjusted by moving the laser spot position on the sample surface. More specifically, the wedge direction of the middle cavity is oriented perpendicularly to that of the outer cavities so that the cavity modes can be tuned relative to each other. The samples substrates have been polished to allow transmission experiments.

\section{Experiment}


Figure 2 displays the measured angular resolved emission at $6 \mathrm{~K}$ (sample A) under continuous-wave resonant pumping of the middle energy mode (pump mode of energy $E_{p}$ and wavevector $k_{p}=0$ ) at normal incidence when all cavities are resonant. The strong optical coupling through DBR 1 and 2 results in lifting the degeneracy and the resulting normal optical modes are split by the same energy (as can be seen from the three cavity-like background emission with upward dispersions). Above a threshold power, one observes the apparition of a strong, spectrally narrow emission at the lower energy (signal of energy $E_{s}$ ) as well higher energy (idler of energy $E_{i}$ ). The emission shows the characteristic signature or parametric oscillation with energy $\left(2 E_{p}=E_{s}+E_{i}\right)$ and momentum conservation $\left(2 k_{p}=k_{s}+k_{i}\right)$. These conservation constraints nicely reflect in the off-branch negative dispersion [8] of the idler mode symmetric to the dispersion of the signal mode, in contrast to the positive dispersion of the signal mode. Given the low cavity finesse of this sample (740), the parametric scattering generates photon pairs of different momentum within the mode linewidth. As a result, signal and idler photons can be collected within $+/-7$ degrees. Moreover, as can be seen on Figure 2, the background emission clearly reveals a weak light-matter coupling regime (as opposed to polariton dispersions). In contrast to previously observed parametric oscillation in single microcavities, here, energy conservation and phase-matching is obtained thanks to the triple cavity resonance, without relying on the polariton dispersion $[2,3,4]$. We note however, that this parametric oscillation can also be observed in the strong light-matter coupling regime in the same structures (for low powers, specific detunings and temperatures below $50 \mathrm{~K}$ ).

Figure 3 (a) shows the signal and idler intensity versus pump power (sample B). The pump-power dependence shows the OPO threshold at $2.4 \mathrm{kWcm}^{-2}$. Above threshold, the signal increases linearly, as expected from the theory [9]. On the contrary, the idler mode saturates above threshold partly because this mode is quasi-degenerate with the QW absorption continuum, inducing losses and fast dephasing [6]. Additional saturation mechanism occurs for the idler state as a result of the possible elastic scattering of the idler states on the third branch towards the second or first branch (as discussed in the next section). From a practical point of view for quantum optics applications, it is known that the attenuation of the idler will not reduce the visibility of the photon pair correlations but only the overall coincidence counting rate [10]. Nevertheless, the saturation of the idler beam causes a problem for quantum noise measurements [11].

Figure 3 (b) also shows the emission for a laser spot position on the sample for which all three cavities are not resonant, precluding energy conservation and parametric conversion. Nevertheless, above a higher pump power threshold of $6 \mathrm{~kW} / \mathrm{cm}^{-2}$, the structure then behaves as a conventional VCSEL (vertical cavity surface emitting laser) with a strong emission at the low energy mode (but no idler emission nor energy conservation requirement).

Parametric oscillation in these structures was demonstrated up to at least $150 \mathrm{~K}$ (the samples were optimized for low temperatures only). This surpasses previous systems that rely on strong exciton-photon coupling, limited to about $50 \mathrm{~K}$ (for InGaAs QWs and comparable materials [3]). Here, no intrinsic limitation remains for operation at room temperature. 


\section{Generation of photon pairs with equal intensities and larger angles}

Even though very flexible, the scheme described above still presents some drawbacks for some specific applications. First, the signal and idler photons are emitted close to $0^{\circ}$ and may be found difficult to separate. Second, even though several schemes can be envisioned for reducing the idler saturation above threshold, one may want to access easily twin photon beams with equal intensities (this is for instance necessary for quantum optics applications relying on noise measurements [11]). Below, we discuss a different type of approach that solves these problems, while giving further insight into the physics of the triple microcavity structures.

As opposed to the vertical inelastic inter-branch parametric scattering discussed above, we now focus on horizontal elastic inter-branch scattering, where the energy of the signal and idler beams are unchanged as compared to the pump beam. This is sketched in Figure 4 (a): the pump beam still excites the middle-energy cavity mode and we consider the scattering towards the low-energy cavity mode. In order to separate the different possible scattering mechanisms, the position of the laser spot on the sample is set to correspond to a detuning for which the phase-matching for the vertical parametric scattering is forbidden (the three cavities are now slightly off-resonances so that the three coupled modes are not split by the same energy). The emission is again measured in transmission and the Fourier plane is imaged onto a CCD camera in order to measure the emission along both $\theta_{x}$ and $\theta_{y}$ angles $(\mathrm{O} z$ being the growth axis). Figure 4 (b) shows the result of an excitation for a large negative detuning (the pump mode lies $10 \mathrm{meV}$ below the QW excitonic energy), where all non-linear scattering mechanisms are weak or suppressed. In this case, the emission shows a strong contribution of resonant Rayleigh scattering along the crystal axis (horizontal and vertical directions in the figure) and weaker Rayleigh scattering along other directions such as the one indicated by the dotted arrow. A weak Rayleigh ring is visible in the background. The interpretation in terms of disorder-induced Rayleigh scattering is confirmed by the pump-power linear dependence, shown in Figure 5 (a). Interestingly, in previously reported resonant Rayleigh scattering experiments on microcavities $[11,12]$, the resonant excitation had to be performed along some direction that does not respect the symmetry of the planar cavity. As a result, the Rayleigh scattering mostly reveals a ring, independently of any preferred direction in the system. On the contrary, our system allows a resonant excitation along a high symmetry axis of the system, which allows to clearly distinguish specific directions favored by the structural disorder. In particular, on observes mostly the contributions of the two perpendicular crystal axis, but also other directions at specific angles $\left(0^{\circ}, 30^{\circ}, 45^{\circ}, 60^{\circ}, 90^{\circ} \ldots\right)$. The disorder induced in the DBRs by the small but non-negligible lattice mismatch between GaAs and AlAs is known to result in preferred directions in the sample plane, but we believe that the triple microcavity structures reveals to be a very convenient system to study this problem.

One now focuses on detunings close to zero, for which large non-linearities are expected. Figure 4 (c) and (d) show the image of the Fourier plane when exciting at high power (above $100 \mathrm{~mW}$ ), for detunings close to zero, on two slightly different spots on the sample. Aside the strong scattering along the horizontal and vertical directions, one also observe a stronger emission along other directions. The analysis of the pump-power dependence shows that these directions are subject to parametric scattering. This is clearly 
visible in Figure 5 (b) that shows a quadratic power dependence for intermediate powers, followed by a threshold-like behavior. One concludes that the parametric scattering preferentially occurs in (some of) the directions that favor Rayleigh scattering (as can be observed when the non-linearities are inefficient, i.e. at negative detuning or for very low powers). Above the threshold, on recovers the linear dependence expected for the OPO. We remark that the emission angles of the photon pairs is now larger than $+/-15^{\circ}$, allowing an easy spatial separation of the two signal and idler beams. Moreover, the signal and idler beams are intrinsically twin, with the same gain and losses, so that they display exactly the same intensity dependence. In terms of quantum optics applications, this is a very favorable situation for quantum noise measurements [11].

\section{Conclusion}

In conclusion we presented a study of the optical parametric oscillation in a vertical triple microcavity structure (a micro-vertical TROPO). This system displays a high flexibility for generating photon pairs and has many similarities to VCSELs. It is has an ultra-low threshold; it can operate under continuous wave excitation and under injection at normal incidence. Its geometry is easily compatible with an electrical injection. Further prospects concern the operation at room temperature as well as measurements of the emitted photon statistics.

\section{Acknowledgments}

This work was supported by the European Community through the Marie Curie Project “Clermont 2" contract number MRTN-CT-2003-503677. 


\section{REFERENCES}

1. N. Gisin, G. Ribordy, W. Tittel and H. Zbinden, Rev. Mod. Phys. 74 (2002) 145.

2. P. G. Savvidis, J.J. Baumberg, R.M. Stevenson, M.S. Skolnick, D.M. Whittaker and J.S. Roberts, Phys. Rev. Lett. 84 (2000) 1547.

3. M. Saba et al., Nature 414 (2001) 731

4. G. Dasbach et al. Phys. Rev. B 71, (2005) 161308(R).

5. C. Diederichs and J. Tignon, App. Phys. Lett. 87 (2005) 251107.

6. C. Diederichs, J. Tignon, G. Dasbach, C. Ciuti, A. Lemaître, J. Bloch, Ph. Roussignol and C. Delalande, Nature 440 (2006) 904.

7. J. Tignon, C. Ciuti, G. Dasbach and C. Diederichs, French Patent Application No 05/05 708 (06 June 2004).

8. P.G. Savvidis et al., Phys. Rev. B 64 (2001) 075311.

9. D.M. Whittaker, Phys. Rev. B 63 (2001) 193305.

10. Z.Y. Ou and L. Mandel, Phys. Rev. Lett. 62 (1989) 2941.

11. M. Romanelli, C. Leyder, J.-P. Karr, E. Giacobino and A. Bramati, cond-mat/0505639 (2005); M. Romanelli, C. Leyder, J.-P. Karr, E. Giacobino and A. Bramati, J. Opt. B: Quantum Semiclass. Opt. 7 (2005) S523-S531.

12. R. Houdré, C. Weisbuch, R.P. Stanley, U. Oesterle and M. Ilegems, Phys. Rev. B 61 (2000) R13333. 


\section{Figure 1}

Triply resonant semiconductor microcavity consisting of : a GaAs substrate, three $\lambda$-GaAs cavities coupled through Bragg mirrors DBR1 and DBR2 and enclosed by a bottom and top DBR. The mirrors are made of pairs of $\lambda / 4$ layers of GaAs and AlAs. Each cavity contains one or several InGaAs QWs.

\section{Figure 2}

Angle-resolved emission measured in transmission (sample A). $\mathrm{T}=6 \mathrm{~K}$. Parametric oscillation under resonant excitation of the second mode above OPO threshold. The detuning of the pump mode with respect to the QW exciton energy is $+10 \mathrm{meV}$. The idler emission is multiplied by 200 .

\section{Figure 3}

(a) Pump-power dependence of the optical parametric oscillator (sample B). Emitted intensity for the signal mode (open circles) and idler (closed circles). (b) The open triangles indicate the emission at the lowest-energy mode for an excitation of the middle mode and for a position on the sample surface for which energy conservation does not allow parametric conversion. In this case, the system behaves as a regular VCSEL laser with a higher threshold.

\section{Figure 4}

(a) Scheme of the horizontal scattering mechanism (resonant Rayleigh scattering or parametric scattering). The pump beam excites resonantly the middle-energy branch. (b) Resonant Rayleigh scattering for large negative detuning (- $10 \mathrm{meV})$. The dotted arrow shows one of the (weak) scattering directions out of the vertical or horizontal crystal axis. (c) and (d) Examples of parametric scattering (detuning close to 0 in both cases).

\section{Figure 5}

Pump-power dependence of the emission for the horizontal scattering mechanism. (a) Strong negative detuning (- $10 \mathrm{meV})$. (b) 0 detuning. 


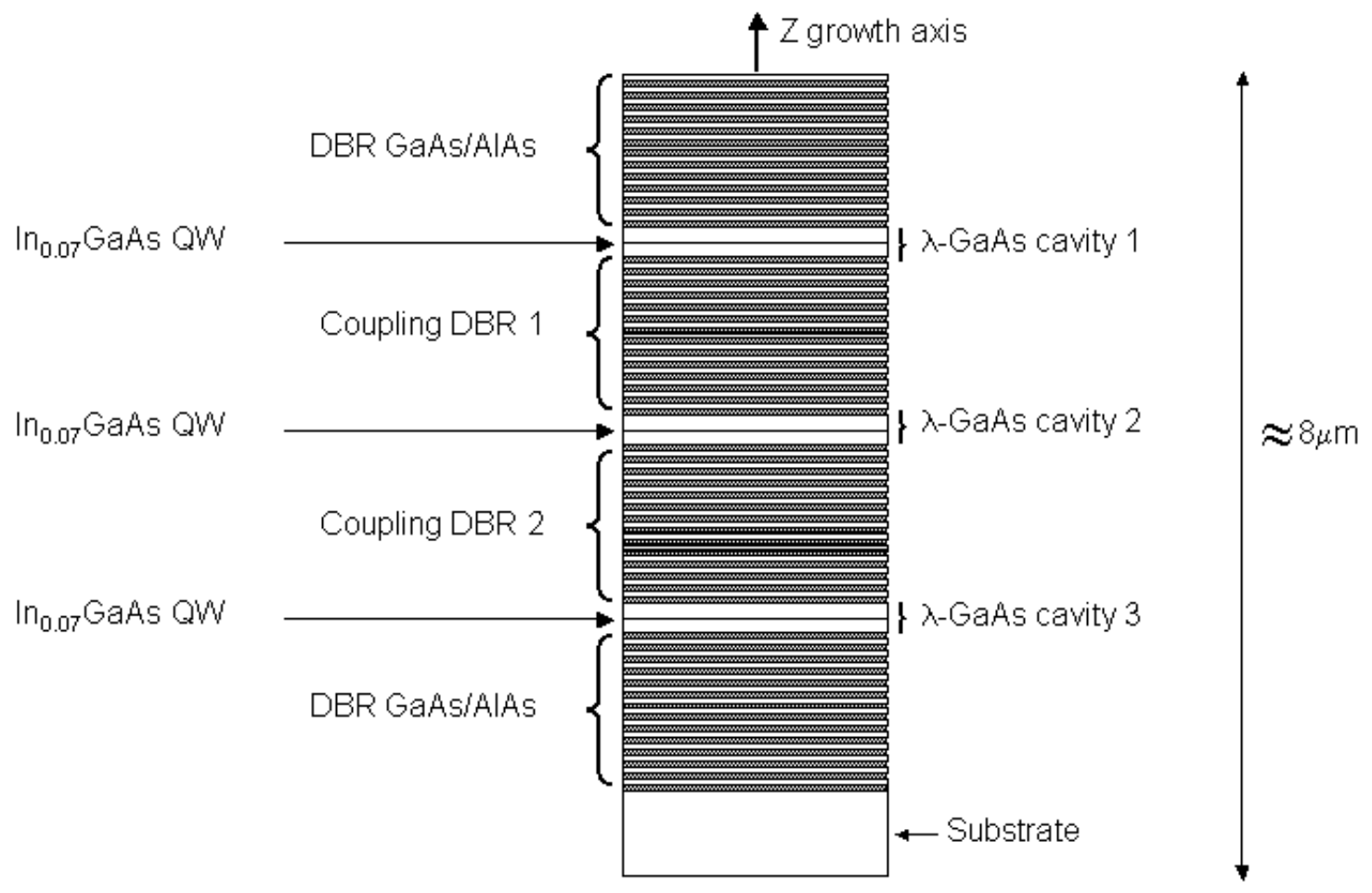

Figure 1 


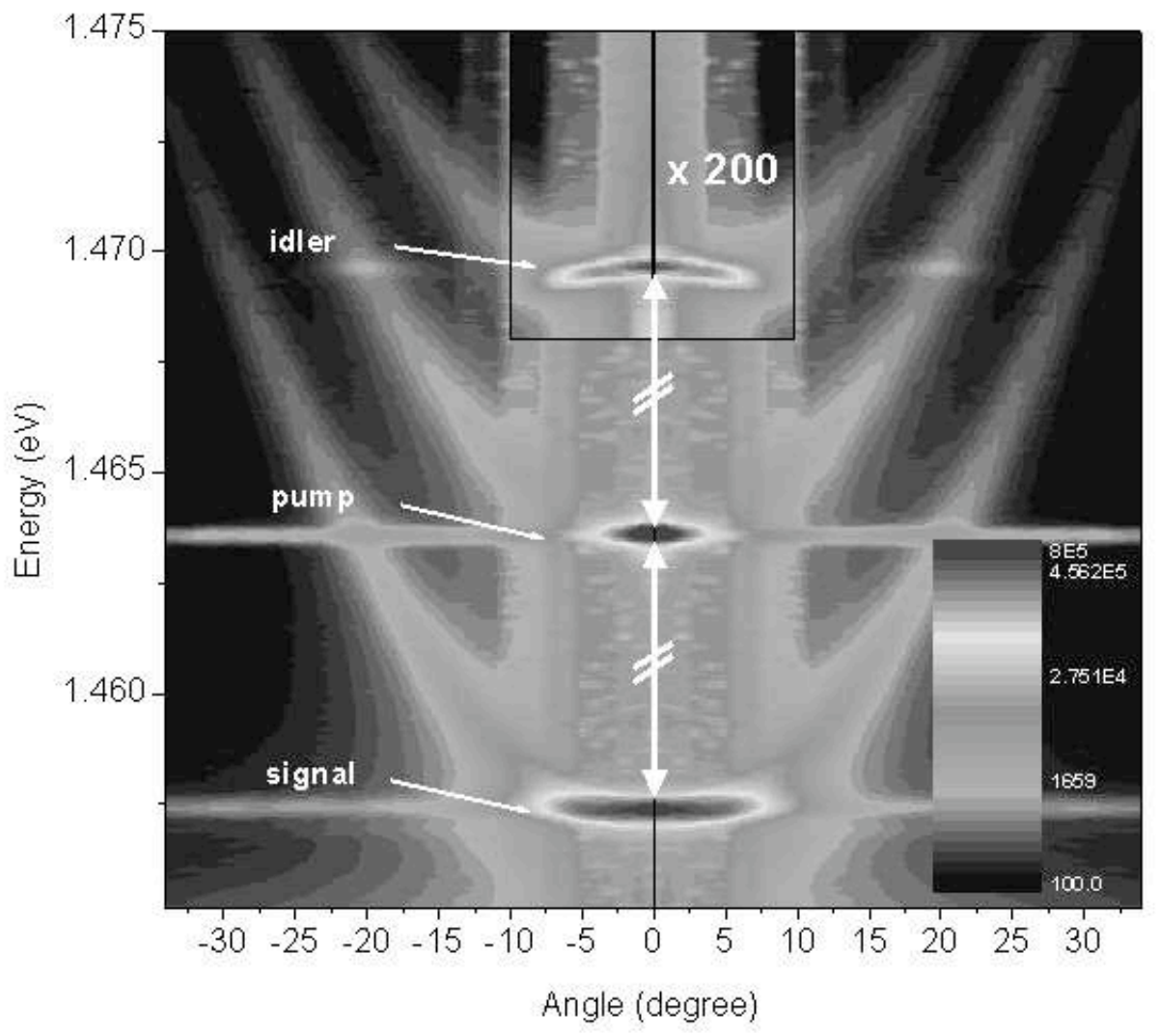

Figure 2 


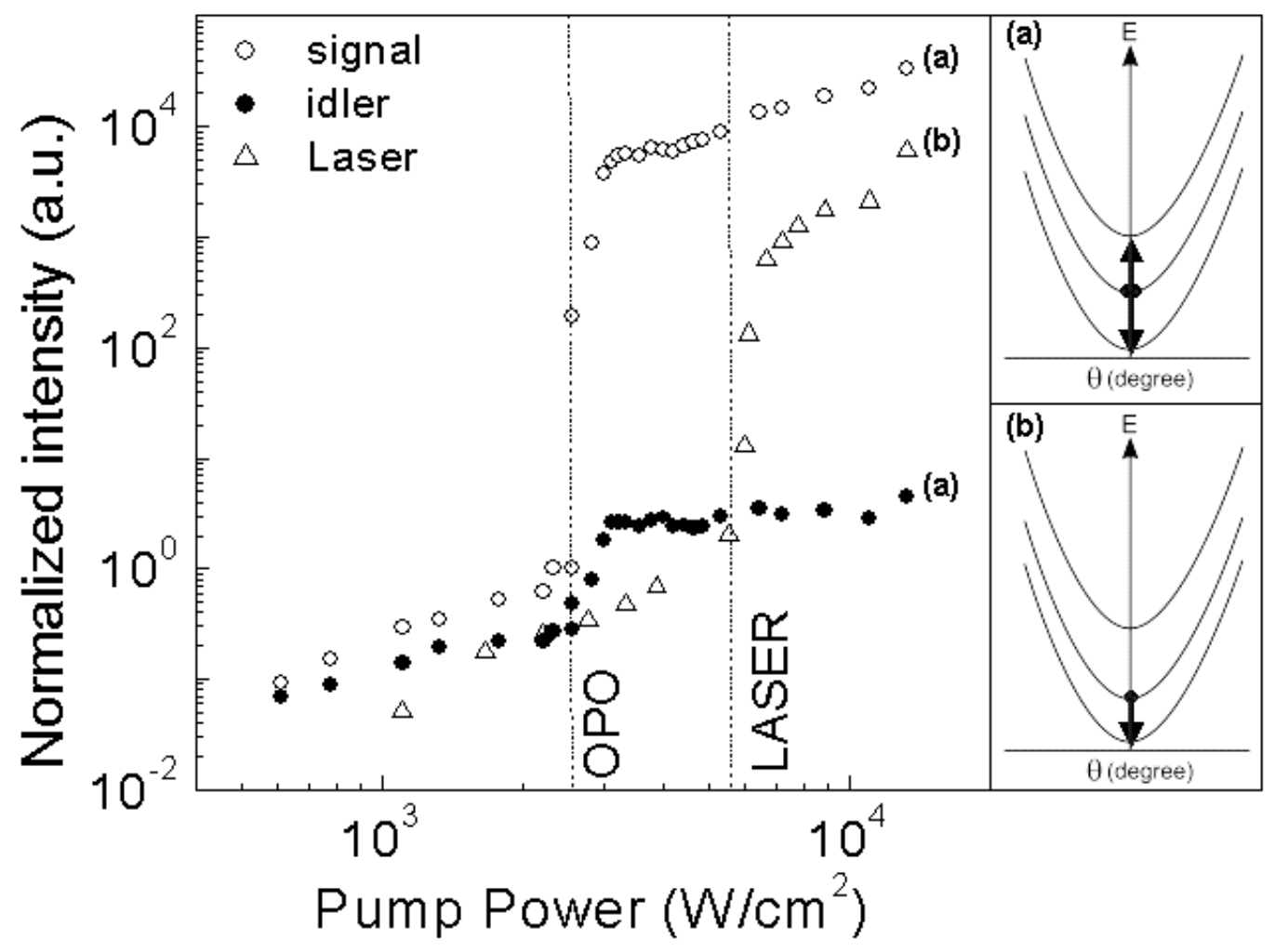

Figure 3 

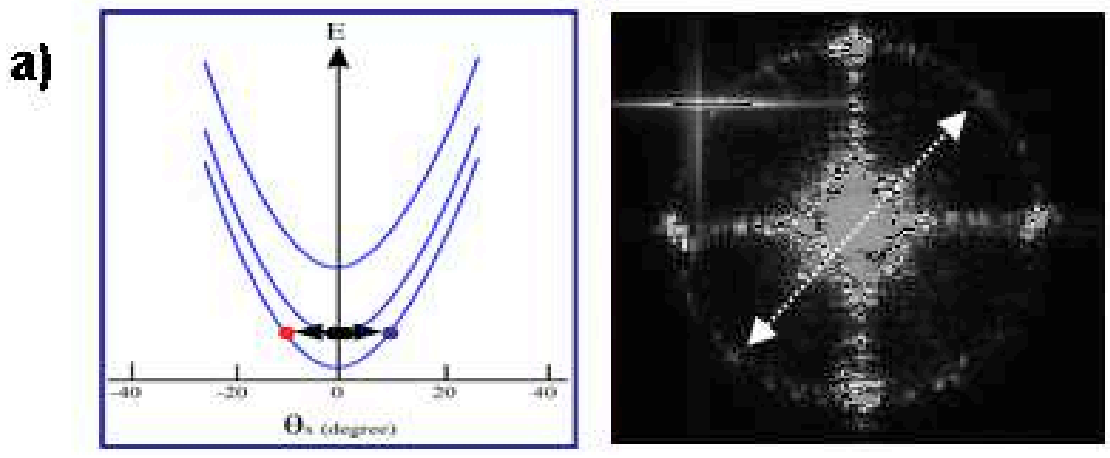

b)
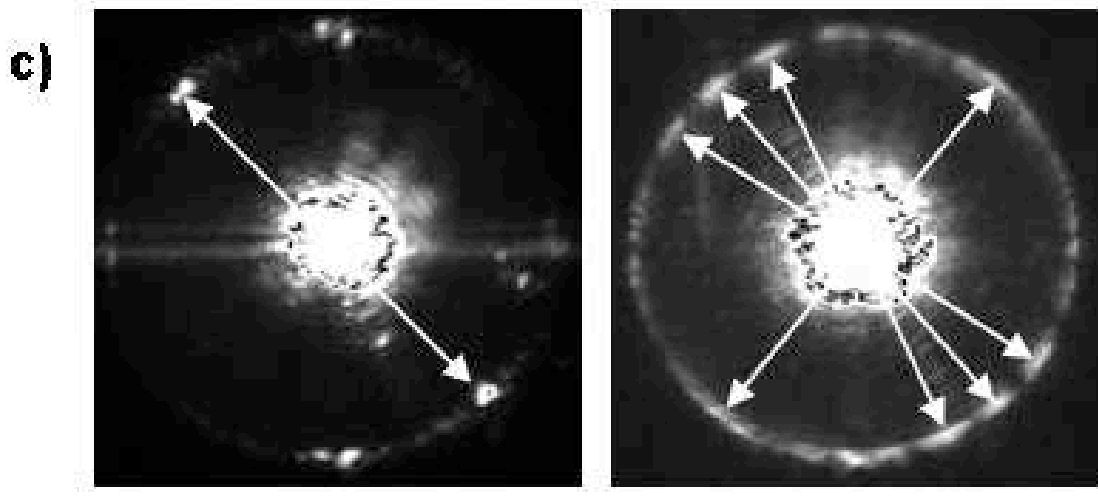

d)

Figure 4 

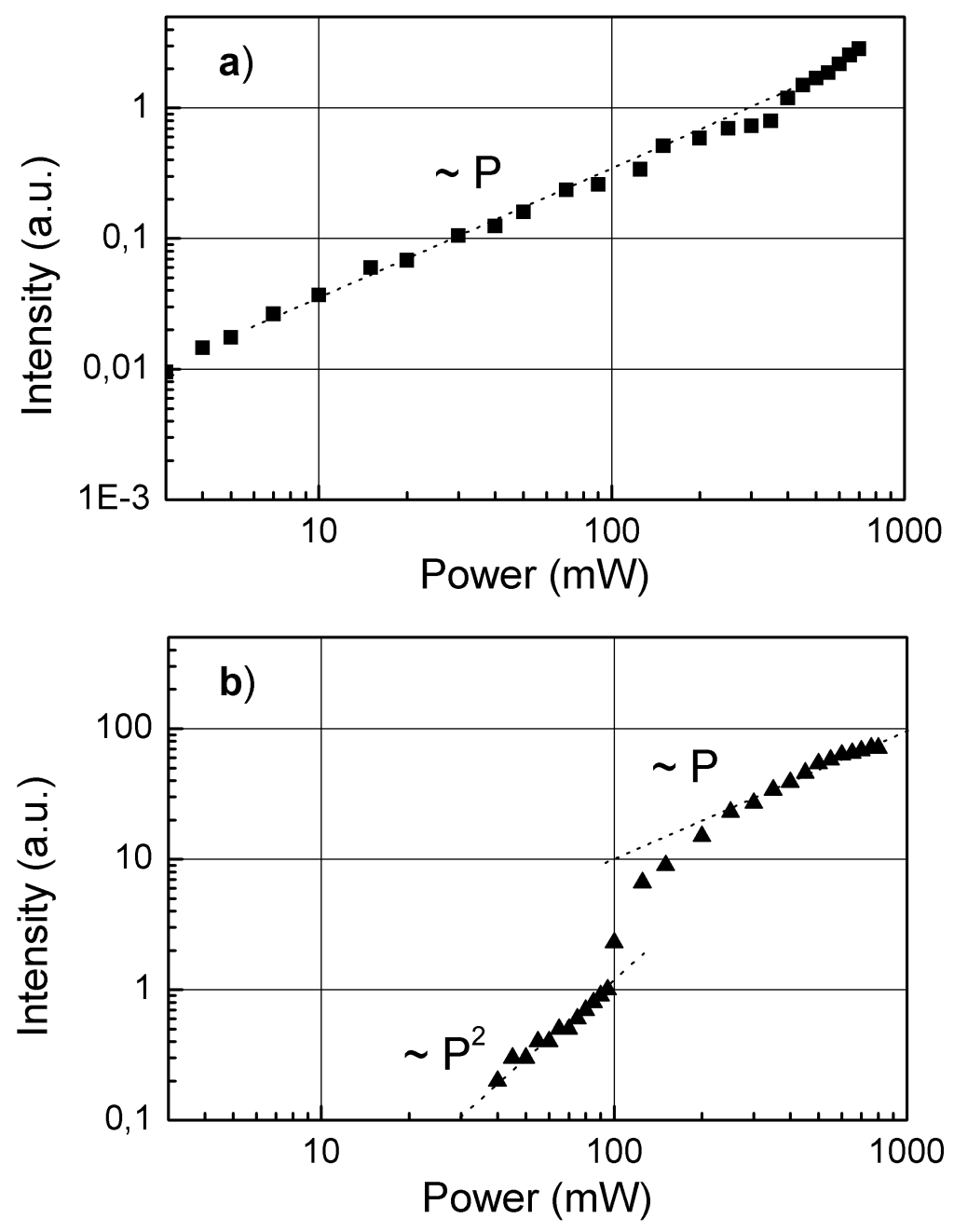

Figure 5 